\title{
Effect of nucleating agents on crystallization kinetics of PET
}

\author{
X. L. Jiang*1, S. J. Luo' ${ }^{1}$ K. Sun ${ }^{1}$, X. D. Chen ${ }^{2}$ \\ ${ }^{1}$ State Key Laboratory of Metal Matrix Composites, Shanghai Jiao Tong University, Shanghai 200030, China \\ ${ }^{2}$ Shanghai Sunny New Technology Development Co. Ltd., Shanghai, 201108, China
}

Received 27 January 2007; accepted in revised form 7 April 2007

\begin{abstract}
Effects of three nucleating agents concluding talc, sodium benzoate (SB) and an ionomer (Ion., $\mathrm{Na}^{+}$) on crystallization of poly(ethylene terephthalate) (PET) were studied by differential scanning calorimetry (DSC) and polarized optical microscope (POM), the parameters of crystallization kinetics were obtained through Avrami and Ozawa equations. The fold surface free energy $\sigma_{e}$ of pure PET and PET/nucleating agent blends was calculated by Hoffman-Lauritzen theory. The results indicate that the three kinds of nucleating agents increase the crystallization rate constant through promoting their nucleating effect for PET crystallization, among which SB is the best one with the same content. The crystallization mode of PET might shift from three-dimensional growth to two-dimensional growth by the addition of the nucleating agents. The values of $\sigma_{e}$ of PET/nucleating agent blends are much less than that of pure PET, and PET/SB (99:1) blend has the least value of $\sigma_{e}\left(18.2 \mathrm{~mJ} / \mathrm{m}^{2}\right)$. The conclusion based on Hoffman theory is similar to the analysis by Avrami and Ozawa equations.
\end{abstract}

Keywords: thermal properties, poly(ethylene terephthalate) (PET), nucleating agents, crystallization kinetics

\section{Introduction}

Poly(ethylene terephthalate) (PET) has already been widely used in the production of fiber, film, bottles and engineering plastics, which derives from its good balance of thermal and mechanical properties. However, it has not gained noticeable application in the field of injection molding because of its low crystallization rate. In order to accelerate the crystallization rate of PET and obtain the desired morphology and properties, a great deal of effort has been made into studying the crystallization kinetics corresponding to the change of the performed properties [1-9].

It is generally considered that the addition of nucleating agents gives rise to increase the crystallization rate of PET [10-13]. A number of suggestions have been reported to add different substances acting as nucleating agents to PET in order to improve its applied or processing properties. The additives exert an influence on the rate of the crystallization process, its morphology, the spherulite size and its distribution and so the physical properties of final product [14-16].

The nucleating agents can be mainly divided into three kinds: inorganic additives, organic additives and polymers, the nucleating effect on PET crystallization depends on several aspects, such as the size and geometry of the particles, the surface structure and interfacial interactions with the polymer matrix [10-12]. A lot of study has discussed the effect of a single nucleating agent on the crystallization of PET, but this paper focuses on the effect of three kinds of nucleating agents on the crystallization kinetics of PET, such as talc, sodium benzoate (SB) and an ionomer (Ion., $\mathrm{Na}^{+}$) which belongs to inorganic additives, organic additives and polymers, respectively. Based on Hoffman-Lauritzen theory, 
the fold surface free energy $\sigma_{e}$, of PET/nucleating agent blends are compared with that of pure PET.

\section{Experimental}

\subsection{Materials}

Poly(ethylene terephthalate) (PET) with the intrinsic viscosity of $0.80 \mathrm{dl} \cdot \mathrm{g}^{-1}$ was produced by Jinshan Petroleum Chemistry Company (Shanghai, China). The nucleating agents were included talc, sodium benzoate (SB) and an ionomer $\mathrm{Na}^{+}$. The talc (800 mesh) was supplied by Haicheng Company (Zhejiang, China). The sodium benzoate with analytically pure was supplied by Shanghai Chemical Reagent Company (Shanghai, China). The ionomer $\mathrm{Na}^{+}$(Surlyn 8920) with the melting point of $88^{\circ} \mathrm{C}$ was supplied by DuPont Company (the United States). Figure 1 shows the chemical structure of the ionomer $\mathrm{Na}^{+}$.

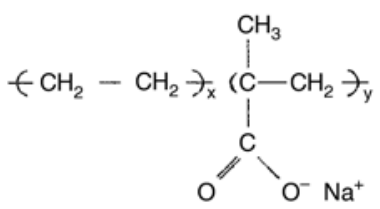

Figure 1. The chemical structure of the ionomer $\mathrm{Na}^{+}$

\subsection{Sample preparation}

Before the blending, PET was dried in a vacuum oven at $80^{\circ} \mathrm{C}$ for $10 \mathrm{~h}$. The talc, the sodium benzoate, and the ionomer $\mathrm{Na}^{+}$were all dried under vacuum at $60^{\circ} \mathrm{C}$ for $10 \mathrm{~h}$. PET was mixed with three kinds of nucleating agents above in the chamber of a Haake Rheometer RC90 (Germany) at $265^{\circ} \mathrm{C}$ and $80 \mathrm{rpm}$, respectively. The mix process lasted about $5 \mathrm{~min}$, the composition was moved out and used for all differential scanning calorimetry (DSC) studied on crystallization kinetics.

\subsection{DSC analysis}

The crystallization behavior was analyzed with a PerkinElmer Paris 1 DSC instrument in a nitrogen atmosphere. The specimens were weighted in the range 5 to $6 \mathrm{mg}$. Overall crystallization rate studies were carried out on completely amorphous samples. For the cases of nonisothermal crystallization, the samples were heated to $265^{\circ} \mathrm{C}$ at a rate of $10^{\circ} \mathrm{C} / \mathrm{min}$ and held for $5 \mathrm{~min}$ to erase all previous thermal history. The first cooling runs at rates of
$5,10,15$ and $20^{\circ} \mathrm{C} / \mathrm{min}$ from $265^{\circ} \mathrm{C}$ to room temperature were recorded.

For isothermal crystallization experiments, the samples were heated to $265^{\circ} \mathrm{C}$ at a rate of $10^{\circ} \mathrm{C} / \mathrm{min}$ and held for $5 \mathrm{~min}$ to erase all previous thermal history, then cooled rapidly $\left(100^{\circ} \mathrm{C} / \mathrm{min}\right)$ to the proposed crystallization temperature $\left(T_{c}\right)$ and maintained at that temperature for the time necessary for the complete crystallization of pure PET and PET/nucleating agent blends. The enthalpy evolved during the isothermal crystallization was recorded as a function of time at different $T_{c}$ 's After crystallization, the samples were heated to $265^{\circ} \mathrm{C}$ at a rate of $10^{\circ} \mathrm{C} / \mathrm{min}$. The melting temperature $\left(T_{m}\right)$ and enthalpy of fusion $\left(\Delta H_{f}\right)$ values of the composites were calculated from the maximum and the area under the endothermic peak, respectively.

\section{Results and discussion}

\subsection{The isothermal crystallization kinetics of PET based on Avrami equation}

The crystallization kinetics of polymers under isothermal conditions for various modes of nucleation and growth can be well approximated by the known Avrami equation [17, 18]. The general form of the Equation (1) is:

$X_{t}=1-\exp \left(-K_{t} t^{n}\right)$

where $X_{t}$ is the relative crystallinity at different crystallization times, $n$ is a constant depending on the mechanism of nucleation and the form of crystal growth, and $K_{t}$ is crystallization rate constant related to nucleation and growth parameters. $X_{t}$ can be calculated according to Equation (2):

$X_{t}=\frac{Q_{t}}{Q_{\infty}}=\frac{\int_{0}^{t} \frac{\mathrm{d} H}{\mathrm{~d} t} \mathrm{~d} t}{\int_{0}^{\infty} \frac{\mathrm{d} H}{\mathrm{~d} t} \mathrm{~d} t}$

where $Q_{t}$ and $Q_{\infty}$ are the amounts of enthalpy generated at time $t$ and infinite time $t_{\infty}$, respectively, and $\mathrm{d} H / \mathrm{d} t$ is the enthalpy evolution rate.

The Avrami equation can be written as follows (3):

$\ln \left[-\ln \left(1-X_{t}\right)\right]=n \ln t+\ln K_{t}$

From a graphic representation of $\ln \left[-\ln \left(1-X_{t}\right)\right]$ versus $\ln t, n$ (the slope of the straight line) and the 

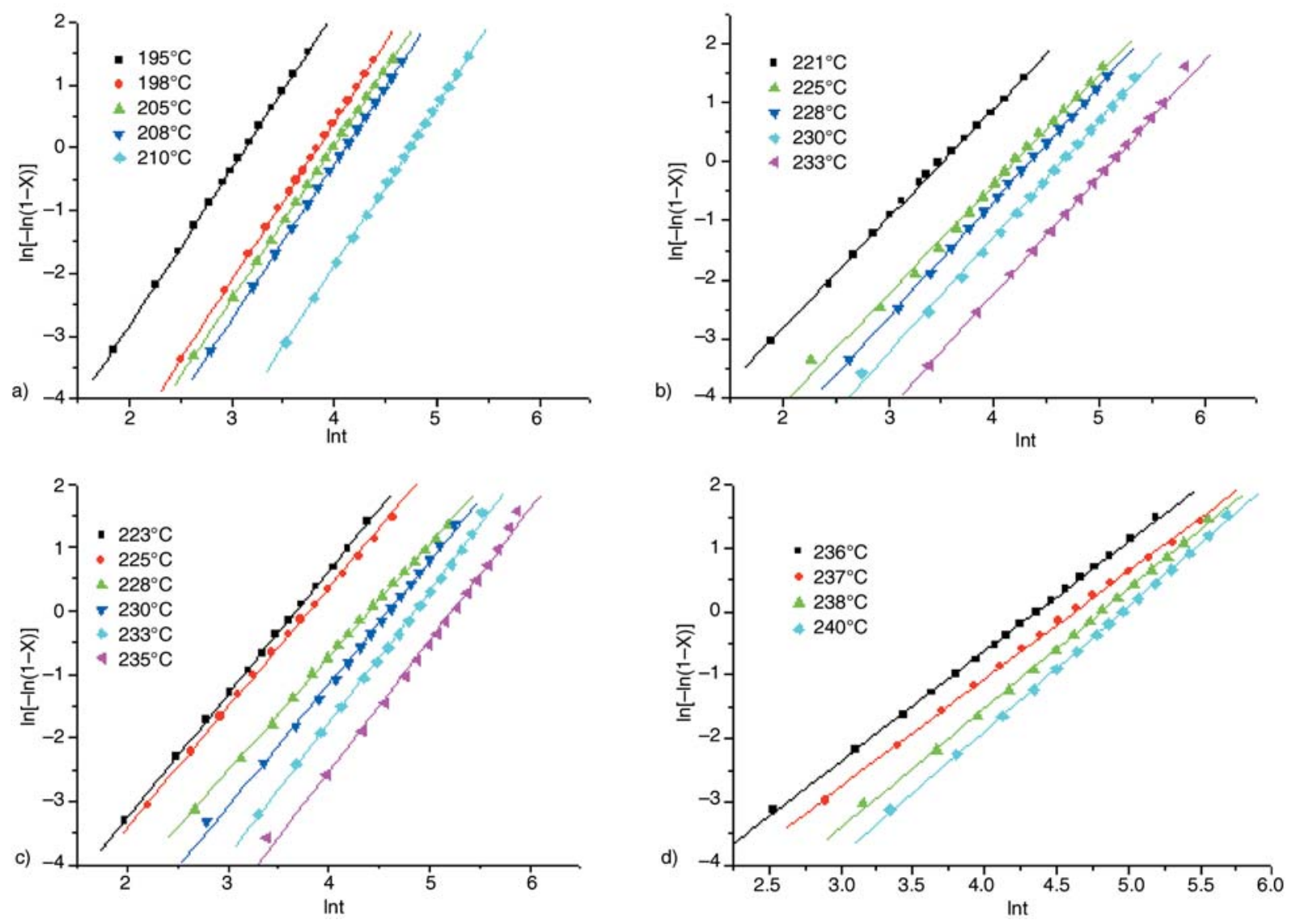

Figure 2. Plots of $\ln \left[-\ln \left(1-X_{t}\right)\right]$ versus $\ln t$ for isothermal crystallization of pure PET and PET/nucleating agent blends.

(a) pure PET; (b) PET/talc = 99:1; (c) PET/Ion., Na+ = 99:1; (d) PET/SB = 99:1

crystallization rate constant $K_{t}$ (the intersection with the $y$ axis) can be obtained. The plots of $\ln \left[-\ln \left(1-X_{t}\right)\right]$ versus $\ln t$ are shown in Figure 2 and the parameters are listed in Table 1. As shown in Table 1, the crystallization rate constant $K_{t}$ of pure PET and PET/nucleating agent blends decreases with increasing temperature. $K_{t}$ of PET/nucleating agent blends is larger than that of pure PET, and PET/SB (99:1) blend has the largest $K_{t}$. The results show that three nucleating agents can increase the crystallization rate of PET, the nucleating effect of the ionomer $\mathrm{Na}^{+}$is slightly better than that of the talc, SB has the most excellent nucleating effect for the crystallization of PET according to the same content of nucleating agent.

Generally, for most of crystallization polymers, the value of Avrami exponent $n$ was found to vary between 1 and 4, corresponding to various growth forms from rod-like to sphere-like [4, 19]. The Avrami model lumps together the formation of nuclei and their subsequent growth. As shown in Table 1, the Avrami exponent $n$ for pure PET is close to 2.5 , it shows that the crystallization mode is a three-dimensional growth of nuclei [20]. While
Table1. Parameters of isothermal crystallization from Avrami equation $\left(T_{c}\right.$ : the crystallization temperature, $K_{t}$ : the crystallization rate constant, n: the Avrami exponent, $t_{1 / 2}$ : the crystallization halftime)

\begin{tabular}{|c|c|c|c|c|}
\hline Samples & $\begin{array}{c}\mathbf{T}_{\mathbf{c}} \\
{\left[{ }^{\circ} \mathbf{C}\right]}\end{array}$ & $\mathbf{K}_{\mathbf{t}}$ & $\mathbf{n}$ & $\begin{array}{c}\mathbf{t}_{\mathbf{1} / 2} \\
{[\mathbf{s}]}\end{array}$ \\
\hline \multirow{5}{*}{ Pure PET } & 195 & $3.841 \cdot 10^{-4}$ & 2.51 & 49.8 \\
\cline { 2 - 5 } & 198 & $6.086 \cdot 10^{-5}$ & 2.53 & 100.2 \\
\cline { 2 - 5 } & 205 & $5.739 \cdot 10^{-5}$ & 2.45 & 120.0 \\
\cline { 2 - 5 } & 208 & $3.949 \cdot 10^{-5}$ & 2.47 & 150.6 \\
\cline { 2 - 5 } & 210 & $5.179 \cdot 10^{-6}$ & 2.57 & 234.6 \\
\hline \multirow{5}{*}{ PET/talc = 99:1 } & 221 & $1.503 \cdot 10^{-3}$ & 1.85 & 27.0 \\
\cline { 2 - 5 } & 225 & $4.062 \cdot 10^{-4}$ & 1.86 & 55.2 \\
\cline { 2 - 5 } & 228 & $2.035 \cdot 10^{-4}$ & 1.96 & 63.0 \\
\cline { 2 - 5 } & 230 & $1.104 \cdot 10^{-4}$ & 1.96 & 87.0 \\
\cline { 2 - 5 } & 233 & $4.111 \cdot 10^{-5}$ & 1.97 & 139.2 \\
\hline PET/Ion., Na+ ${ }^{+}$99:1 & 223 & $7.916 \cdot 10^{-4}$ & 1.94 & 32.4 \\
\cline { 2 - 5 } & 225 & $7.808 \cdot 10^{-4}$ & 1.88 & 36.6 \\
\cline { 2 - 5 } & 228 & $3.796 \cdot 10^{-4}$ & 1.78 & 66.0 \\
\cline { 2 - 5 } & 230 & $1.421 \cdot 10^{-4}$ & 1.93 & 83.4 \\
\cline { 2 - 5 } & 233 & $4.130 \cdot 10^{-5}$ & 1.98 & 109.2 \\
\cline { 2 - 5 } & 235 & $2.066 \cdot 10^{-5}$ & 1.96 & 160.8 \\
\hline PET/SB = 99:1 & 236 & $5.097 \cdot 10^{-4}$ & 1.74 & 63.6 \\
\cline { 2 - 5 } & 237 & $3.878 \cdot 10^{-4}$ & 1.70 & 80.4 \\
\cline { 2 - 5 } & 238 & $1.168 \cdot 10^{-4}$ & 1.88 & 101.4 \\
\cline { 2 - 5 } & 240 & $5.797 \cdot 10^{-5}$ & 1.97 & 118.8 \\
\hline
\end{tabular}


the values of $n$ for PET/nucleating agent blends are found to below 2 . This indicates that the crystallization mode of PET might shift to two-dimensional growth with heterogeneous nucleation [20]. The result shows that the crystallization mode of PET has been changed by the addition of nucleation agents.

The crystallization half-time $t_{1 / 2}$ is defined as the time at which the extent of crystallization is completed $50 \%$. The shorter the half-time, the faster the crystallization rate. It can be determined from the measured kinetic parameters [21-22] (4):

$t_{1 / 2}=\left(\frac{\ln 2}{K_{t}}\right)^{1 / n}$

The value of $t_{1 / 2}$ is listed in Table 1. $t_{1 / 2}$ increases with increasing crystallization temperature $T_{c}$. A higher crystallization temperature leads to a lower supercooling degree, the nuclei for the crystallization are more difficult to form.
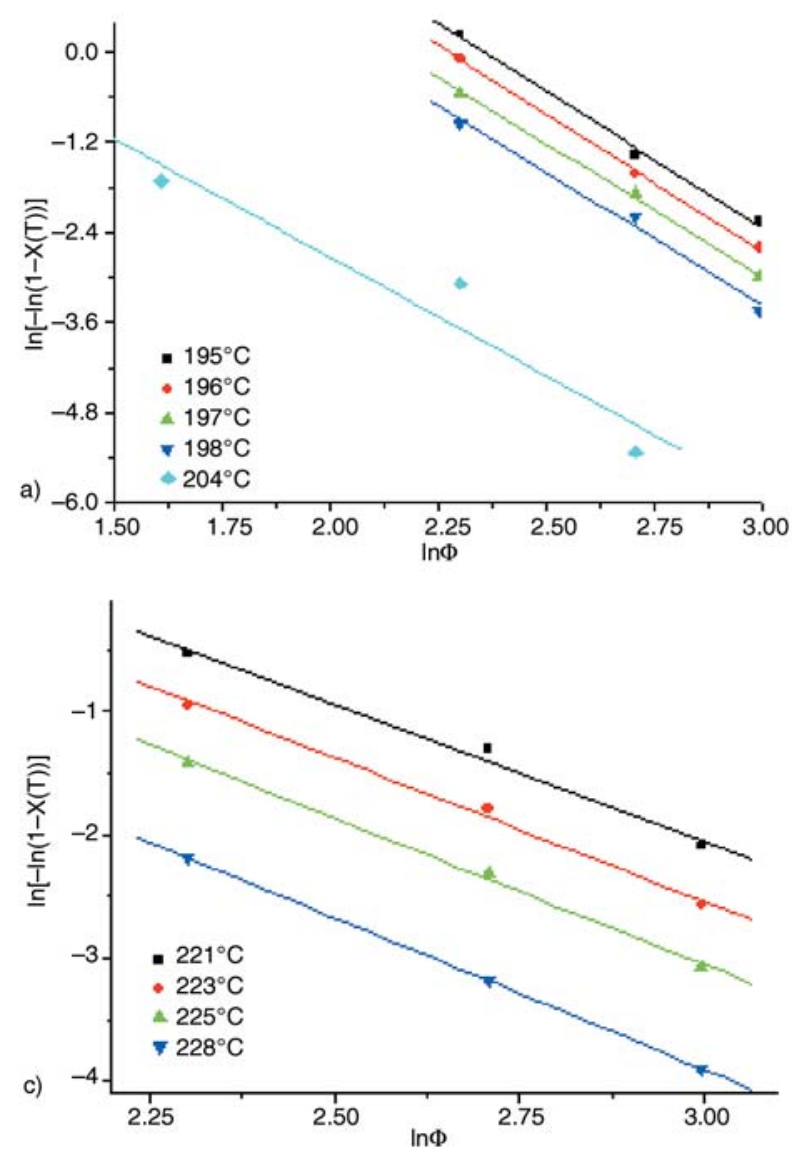

\subsection{The nonisothermal crystallization kinetics of PET based on Ozawa equation}

There are several modes for the study of nonisothermal crystallization kinetics of polymers. The Avrami equation can be directly used to study nonisothermal crystallization, but it didn't take into account the effect of the constant cooling on the crystallization, so the non-linearity of Avrami plots were often obtained. While accounting for the effect of cooling (or enthalpying) rate $\Phi$ on crystallization from the melt (or the glassy state), Ozawa [23] amended and extended Avrami equation, and the Ozawa equation is (5):

$$
X(T)=1-\exp \left(-\frac{K(T)}{\Phi^{m}}\right)
$$

where $\Phi$ is the cooling rate, $m$ is the Ozawa exponent that depended on the crystal growth and nucleation mechanism and $K(T)$ is the crystallization rate constant.

The Ozawa equation can be written Equation (6).
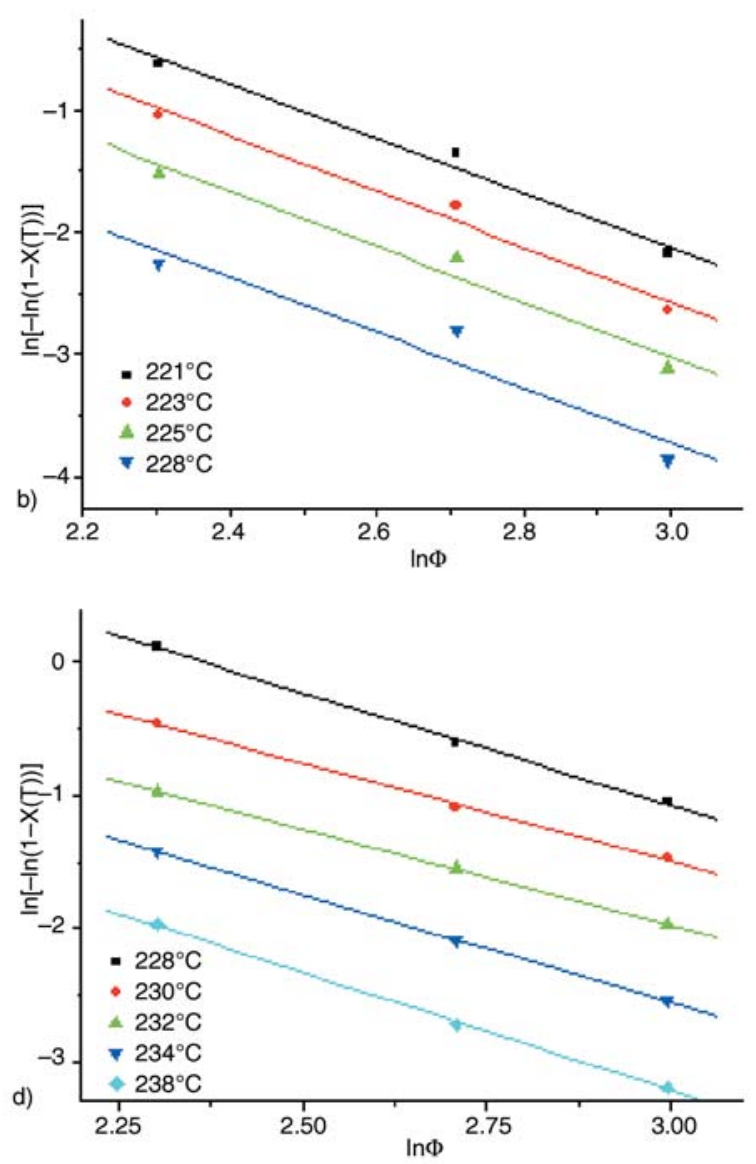

Figure 3. Plots of $\ln \{-\ln [1-X(T)]\}$ versus $\ln \Phi$ for nonisothermal crystallization of pure PET and PET/nucleating agent blends. (a) pure PET; (b) PET/talc = 99:1; (c) PET/Ion., $\mathrm{Na}^{+}=99: 1$; (d) PET/SB = 99:1 
Table 2. Parameters of nonisothermal crystallization from the Ozawa equation ( $T$ : the crystallization temperature, $K(T)$ : the crystallization rate constant, $m$ : the Ozawa exponent)

\begin{tabular}{|c|c|c|c|}
\hline Sample & $\mathbf{T}\left[{ }^{\circ} \mathbf{C}\right]$ & $\mathbf{l n K}(\mathbf{T})$ & $\mathbf{~}$ \\
\hline \multirow{4}{*}{ Pure PET } & 195 & 8.49 & 3.61 \\
\cline { 2 - 4 } & 196 & 8.25 & 3.63 \\
\cline { 2 - 4 } & 197 & 7.53 & 3.50 \\
\cline { 2 - 4 } & 198 & 7.26 & 3.54 \\
\cline { 2 - 4 } & 204 & 3.56 & 3.14 \\
\hline \multirow{4}{*}{ PET/talc = 99:1 } & 221 & 4.50 & 2.21 \\
\cline { 2 - 4 } & 223 & 4.24 & 2.27 \\
\cline { 2 - 4 } & 225 & 3.78 & 2.27 \\
\cline { 2 - 4 } & 228 & 3.01 & 2.25 \\
\hline \multirow{5}{*}{ PET/Ion., Na+ ${ }^{+}=99: 1$} & 221 & 4.64 & 2.23 \\
\cline { 2 - 4 } & 223 & 4.45 & 2.33 \\
\cline { 2 - 4 } & 225 & 4.09 & 2.38 \\
\cline { 2 - 4 } & 228 & 3.48 & 2.41 \\
\hline \multirow{3}{*}{ PET/SB = 99:1 } & 228 & 3.99 & 1.69 \\
\cline { 2 - 4 } & 230 & 2.93 & 1.47 \\
\cline { 2 - 4 } & 232 & 2.35 & 1.44 \\
\cline { 2 - 4 } & 234 & 2.30 & 1.62 \\
\cline { 2 - 4 } & 236 & 2.09 & 1.77 \\
\hline
\end{tabular}

$\ln \{-\ln [1-X(T)]\}=\ln K(T)-m \ln \Phi$

According to Equation (6), $\ln \{-\ln [1-X(T)]\}$ against $\ln \Phi$ is plotted in Figure 3. The Ozawa plots of linear relationship between and $\ln \Phi$ shows that the Ozawa equation can be priority to explain the nonisothermal crystallization behavior of pure PET and PET/nucleating agent blends. The Ozawa parameters, $m$ and crystallization rate constant $K(T)$, can be obtained through the slope and intercept of the fitting for those data shown in Figure 3 and listed in Table 2.

The value of the Ozawa exponent $m$ for pure PET is almost equal to 3 , in good agreement with the results reported by Ozawa [23]. In general, an Ozawa exponent $m=3$ suggests that the mode at the nonisothermal crystallization of PET is of three-dimensional growth with an athermal nucleation mechanism. While the values of $m$ for PET/ nucleating agent blends are below 3 . This indicates that the crystallization mode might change, which also found in isothermal crystallization process.

The crystallization rate constant $K(T)$ of pure PET and PET/nucleating agent blends decreases with increasing temperature. $K(T)$ of PET/nucleating agent blends is higher than that of pure PET. This also indicates that the crystallization rate of PET increases by the addition of the nucleating agent. PET/SB (99:1) blend has the largest value of $K(T)$ at the same temperature. So among three kinds of nucleating agents, SB has the most excellent nucleating effect for PET crystallization. The nucleating effect of Ion., $\mathrm{Na}^{+}$is slightly better than that of the talc which is the same as the analysis of isothermal crystallization.

\subsection{The fold surface free energy based on Hoffman-Lauritzen relationship}

In studying the crystallization kinetics of crystalline polymers, we need to know the equilibrium melting temperature $\left(T_{m}{ }^{0}\right)$ to calculate the degree of supercooling [23]. After crystallization at $T_{c}, T_{m}$ was obtained by the reheating of the samples at a rate of $10^{\circ} \mathrm{C} / \mathrm{min}$. It was possible to calculate $T_{m}{ }^{0}$ by the plotting of $T_{c}$ versus $T_{m}$ and to observe the intersection of this line with another line with a slope equal to $1\left(T_{m}=T_{c}\right)$ [24]. Plots of $T_{m}$ against $T_{c}$ are shown in Figure 4, and the values of $T_{m}{ }^{0}$ are listed in Table 3.

The crystallization rates at different temperatures were expressed in the form of reciprocal of crystallization half-time $t_{1 / 2}$. Using Hoffman-Lauritzen relationship between the radial growth rate of crystal and crystallization temperature based on crystallization regime theory [25], and following Chan and Isayev [26] $\left(1 / t_{1 / 2}\right)$ and $\left(1 / t_{1 / 2}\right)_{0}$ were used to

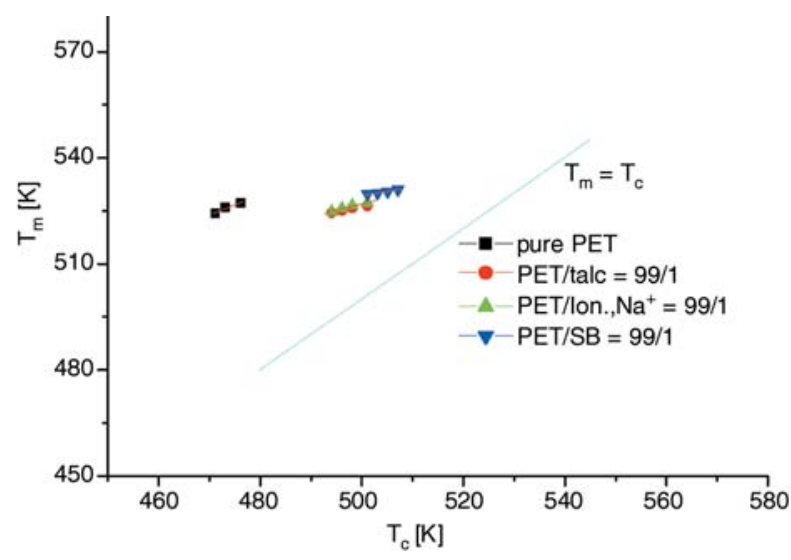

Figure 4. The determination of the equilibrium melting temperature

Table 3. Thermodynamic characteristics of the PET and PET/nucleation agent blends $\left(T_{m}{ }^{0}\right.$ : the equilibrium melting temperature, $\sigma_{e}$ : the fold surface free energy)

\begin{tabular}{|l|c|c|c|c|}
\hline & Pure PET & $\begin{array}{c}\text { PET/talc } \\
(\mathbf{9 9 : 1 )}\end{array}$ & $\begin{array}{c}\text { PET/Ion., Na } \\
\text { (99:1) }\end{array}$ & $\begin{array}{c}\text { PET/SB } \\
(\mathbf{9 9 : 1 )}\end{array}$ \\
\hline$T_{m}{ }^{0}[\mathrm{~K}]$ & 568 & 542 & 541 & 536 \\
\hline$\sigma_{e}\left[\mathrm{~mJ} / \mathrm{m}^{2}\right]$ & 80.3 & 25.4 & 21.3 & 18.3 \\
\hline
\end{tabular}


substitute for the growth rate $G$ and a pre-exponential factor $G_{0}$, respectively. The temperature dependence of the crystallization half life $t_{1 / 2}$ is given by Equation (7).

$$
\frac{1}{t_{1 / 2}}=\left(\frac{1}{t_{1 / 2}}\right)_{0} \exp \left[\frac{-U^{*}}{R\left(T-T_{\infty}\right)}\right] \exp \left[\frac{-K_{g}}{T(\Delta T) f}\right]
$$

where $T$ is the crystallization temperature, $R$ is the universal gas constant, $\Delta T=T_{m}{ }^{0}-T$ is the supercooling, and $f=2 T /\left(T+T_{m}{ }^{0}\right)$ is a correction factor accounting for the reduction in the latent enthalpy of fusion as the temperature decreased, $T_{m}{ }^{0}$ being the equilibrium melting temperature. There are four material constants in Equation (7). (1/t/2) $)_{0}$ is a preexponential factor that includes all terms independent of temperature, $U^{*}$ is the activation energy for the transport of crystallizing units across the phase boundary, $T_{\infty}$ is the temperature below which such transport ceases, and $K_{g}$ is the nucleation parameter. According to Hoffman theory [25], the parameters $U^{*}$ and $T_{\infty}$ are the recommended values of $6284 \mathrm{~J} / \mathrm{mol}$ and $T_{g}-30 \mathrm{~K}$, respectively, $T_{g}$ being the glass transition temperature. Plots of $\ln \left(1 / t_{1 / 2}\right)+$ $U^{*} / R\left(T-T_{\infty}\right)$ against $1 / T(\Delta T) f$ are shown in Figure 5, and the slope of the line is $K_{g}$.

The nucleation parameter $K_{g}$ is given by Equation (8).

$K_{g}=\frac{z b \sigma \sigma_{e} T_{m}^{0}}{\Delta H_{f} k_{B}}$

where $b$ is the monomolecular layer thickness, taken to be the perpendicular separation of (010) planes, this is $5.53 \AA$ [2]. $\sigma$ is the side surface free energy of the polymer crystal, $\sigma_{e}$ is the fold surface free energy, $\Delta H_{f}$ is the enthalpy of fusion per unit

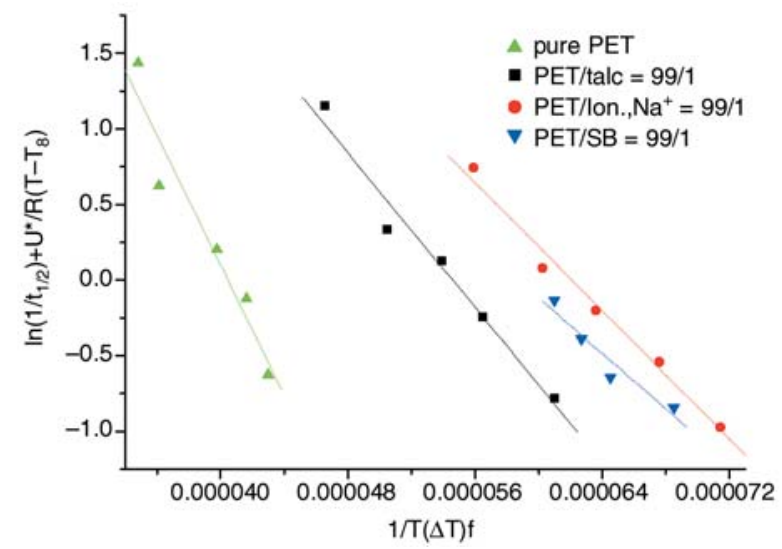

Figure 5. Plots of versus for isothermal crystallization of pure PET and PET/nucleating agent blends volume $\left(2.1 \cdot 10^{8} \mathrm{~J} / \mathrm{m}^{3}\right)[2]$ and $k_{B}$ is the Boltzmann constant equaling to $1.35 \cdot 10^{-23} \mathrm{~J} / \mathrm{mol} \cdot \mathrm{K} . z$ is relationship with crystallization regimes of PET. At high crystallization temperatures (above $490 \mathrm{~K}$ ), corresponding to small degrees of supercooling, regime I kinetics are operative [13]. In this case, surface nucleation involved in crystal growth leads to rapid completion over the surface of the new phase prior to the next nucleation event, and the value of $z$ is 4 . According to the crystallization temperature, the crystallization of PET/nucleating agent blends belongs to this regime. At large degrees of supercooling, i. e. below $490 \mathrm{~K}$, regime II are operative [13], where the rates of the secondary nucleation and spread of the molecular strip along the growing face are comparable, in this case, the value of $z$ is 2 and the crystallization of pure PET belongs to this regime. The side surface free energy $\sigma$ is often estimated as [25] (9):

$\sigma=\alpha \Delta H_{f}\left(a_{0} b_{0}\right)^{1 / 2}$

where $\alpha$ was derived empirically to be 0.11 by analogy with the known behavior of hydrocarbons . The unit cell dimensions, $a_{0}$ and $b_{0}$ for PET used in the analysis are 4.57 and $5.95 \AA$, respectively [2]. Combining Equation (8) and Equation (9), we can observe the Equation (10) as follows:

$K_{g}=\frac{z b \alpha \sigma_{e}\left(a_{0} b_{0}\right)^{1 / 2} T_{m}^{0}}{k_{B}}$

$\sigma_{e}$ of pure PET and PET/nucleating agent blends are determined, and listed in Table 3.Generally speaking, the smaller the fold surface free energy $\sigma_{e}$, the faster the crystallization rate of polymer crystal. As shown in the Table 3 , the value of $\sigma_{e}$ of pure PET is $80.3 \mathrm{~mJ} / \mathrm{m}^{2}$, which is in an agreement with reference [25]. The fold surface free energy $\sigma_{e}$ of PET/nucleating agent blends are obviously less than that of pure PET, and PET/SB (99:1) blend has the least value of $\sigma_{e}\left(18.3 \mathrm{~mJ} / \mathrm{m}^{2}\right)$. The result shows that the conclusion based on Hoffman theory is consistent with the analysis by Avrami and Ozawa equations.

\section{Conclusions}

The effects of three kinds of nucleating agents, including talc, SB and Ion., $\mathrm{Na}^{+}$on the crystallization kinetics of PET were studied by using DSC. 
The parameters of the isothermal and the nonisothermal crystallization kinetics were obtained through Avrami and Ozawa equations, respectively. The results indicate that three nucleating agents can increase the crystallization rate of PET, and SB has the most excellent nucleating effect for the crystallization of PET with the same content of nucleating agent. The crystallization mode of PET might shift from three-dimensional growth to twodimensional growth by the addition of the nucleating agents. The fold surface free energy $\sigma_{e}$ are obtained by using Hoffman-Lauritzen relationship, the values of $\sigma_{e}$ of PET/nucleating agent blends are much less than that of pure PET, and PET/SB (99:1) blend has the least value of $\sigma_{e}\left(18.2 \mathrm{~mJ} / \mathrm{m}^{2}\right)$. The conclusion based on Hoffman theory is consistent with the analysis by Avrami and Ozawa equations.

\section{References}

[1] Li B. Z., Yu J. Y., Lee S., Ree M.: Crystallizations of poly(ethylene terephthalate co ethylene isophthalate). Polymer, 40, 5371-5375 (1999).

[2] Rahman M. H., Nandi A. K.: On the crystallization mechanism of poly(ethylene terepthalate) in its blends with poly(vinylidene fluoride). Polymer, 43, 68636870 (2002).

[3] Kong X. H., Yang X. N., Zhou E., Ma D. H.: Nonisothermal crystallization kinetics of ethylene Terephthalate-ethylene oxide segmented copolymers with two crystallizing segments. European Polymer Journal, 36, 1085-1090 (2000).

[4] Sajkiewicza P., Carpaneto L., Wasiak A.: Application of the Ozawa model to non-isothermal crystallization of poly(ethylene terephthalate). Polymer, 42, 53655370 (2001).

[5] Zhang Z., Ren M., Zhao J., Wu S., Sun H.: Kinetics of non-isothermal cold crystallization of uniaxially oriented poly(ethylene terephthalate). Polymer, 44, 2547-2551 (2003).

[6] Zhang Y.H., Gu L.X.: Study of non-isothermal crystallization kinetics and sequence distribution in poly(ethylene terephthalate-co-isophthalate). European Polymer Journal, 36, 759-765 (2000).

[7] Run M. T., Wu S. Z., Zhang D. Y., Wu G.: Melting behaviors and isothermal crystallization kinetics of poly(ethylene terephthalate)/mesoporous molecular sieve composite. Polymer, 46, 5308-5316 (2005).

[8] Guan B. X., Phillips P. J.: Does isothermal crystallization ever occur? Polymer, 46, 8763-8773 (2005).

[9] Lu X. F., Hay J. N.: Isothermal crystallization kinetics and melting behaviour of poly(ethylene terephthalate). Polymer, 42, 9423-9431 (2001).
[10]. Chen Y., Xu M., Li Y. Y., He J. S.: Effect of nucleating agent and nucleation promoter on the crystallization of polyethylene terephthalate. Acta Polymerica Sinica, 20, 8-14 (1999).

[11] Liu S. L., Ma J. H., Liang B. R.: Study on activity of nucleating agent and promoters for PET. Synthetic Technology and Application, 14, 6-9 (1999).

[12] Guo R. Y., Wei D. F., Lu H.: Effects of nucleating and nucleation promoter on the crystallization of polyethylene terephthalate. Polymer Materials Science and Engineering, 19, 121-124 (2003).

[13] Incarnato L., Motta O., Acierno D.: Thermal behaviour of the PET/Rodrun 3000 system. Polymer, 39, 5085-5091 (1998).

[14] Menczel J., Varga J.: Influence of nucleating agents on crystallization of polypropylene I. talc as a nucleating agent. Journal of Thermal Analysis, 28, 161-174 (1983).

[15] Zhang M. L., Liu Y. L., Zhang X. H.: The effect of elastomeric nano-particle on the mechanical properties and crystallization behavior of polypropylene. Polymer, 43, 5133-5138 (2002).

[16] Saujanya C., Radhakrishnan S.: Structure development and crystallization behaviour of $\mathrm{PP} /$ nano particulate composite. Polymer, 42, 6723-6731 (2001).

[17] Avrami M. J.: Kinetics of phase change-I. General theory. Journal of Chemistry and Physics, 7, 1103 1109 (1939).

[18] Avrami M.: Granulation, phase change and microstructure kinetics of phase change. III. Journal of Chemistry and Physics, 9, 177-184 (1941).

[19] Jabarin S. A.: Crystallization kinetics of polyethylene terephthalate. I. Isothermal crystallization from the melt. Journal of Applied Polymer Science, 34, 85-96 (1987).

[20] Garcia D.: Heterogeneous nucleation of poly(ethylene terephthalate). Journal of Polymer Science: Polymer Physics Edition, 22, 2063-2072 (1984).

[21] Li J., Zhou C. X., Wang G., Tao Y., Liu Q., Li Y.: Isothermal and nonisothermal crystallization kinetics of elastomeric polypropylene. Polymer Testing, 21, 583-589 (2002).

[22] Arroyo M., Lopez-Manchado M. A., Avalos F.: Crystallization kinetics of polypropylene: II. Effect of the addition of short glass fibres. Polymer, 38, 5587-5593 (1997).

[23] Ozawa T.: Kinetics of non-isothermal crystallization. Polymer, 12, 150-156 (1971).

[24] Hoffman J. D., Miller R. L.: Kinetics of crystallization from melt and chain folding in polyethylene fractions revisited: theory and experiment. Polymer, 38, 31513206 (1997).

[25] Hoffman J. D., Davis G. T., Lauritzen J. L.: The rate of crystallization of linear polymers. In: 'Treatise on Solid State Chemistry' (ed.: Hannay N. B.) Plenum Press, New York, Vol. 3, 497-614 (1976).

[26] Chan T. W., Isayev A. I.: Quiescent polymer crystallization: Modeling and measurements. Polymer Engineer and Science, 34, 461-471 (2004). 